\title{
Adaptation and validation of the Portuguese version of Basic Psychological Needs Exercise Scale (BPNESp) to the sport domain and invariance across football and swimming
}

\author{
Diogo Monteiro ${ }^{1,3^{*}}$, Daniel A. Marinho ${ }^{2,3}$, João Moutão ${ }^{1,3}$, Nuno Couto ${ }^{1}$, Raúl Antunes ${ }^{1}$, Luis \\ $\mathrm{Cid}^{1,3}$
}

\begin{abstract}
\end{abstract}
The purpose of this study was to adapt and validate the Basic Psychological Needs Exercise Scale (BPNESp) to the sport domain, and to measure model invariance across football and swimming. Athletes $(n=1382$; 623 football, 759 swimming) with an average age of $18.77 \pm$ SD 7.04 years participated in this study. Results supported the suitability of both the first and second order models, showing that the model was well adjusted to the data. In addition, the model showed the discriminant and convergent validity and composite reliability of the factors, and was invariant between football and swimming ( $\triangle \mathrm{CFI} \leq .01)$.

Keywords: Motivation, basic psychological needs, confirmatory factor analysis, multi-groups analysis

\section{INTRODUCTION}

Self-Determination Theory (SDT: Deci \& Ryan, 1985, 2008), which is a socio-cognitive macro theory on human motivation, is comprised of six mini- theories (cognitive evaluation theory; basic psychological needs theory (BPN); organismic integration theory; causality orientations theory, goal contents theory and relationships motivation theory), with each one systematizing crucial aspects of the motivation scope (Deci \& Ryan, 2000). However, in the present study, only the BPN will be approached. According to the authors (Deci \& Ryan, 2000), the subject's motivation is determined by the satisfactions of three fundamental "nutriments" (i.e., BPN: basic psychological needs): autonomy (i.e., need to feel independent, in that it regulates its own actions), competence (i.e., need to feel capable) and relatedness (i.e., need to relate with others). In accordance with the authors, these three needs, which are innate and universal, can explain how the subjects regulate their own behaviour via the motivational continuum that oscillates between more autonomous or more controlled forms of behaviour regulation. Thus, a social environment that supplies conditions supporting the individuals' autonomy promotes the satisfaction of the three basic BPN and, thereby, results in positive behavioural consequences, such as more intrinsic motivation and wellbeing (Teixeira, Carraça, Markland, Silva, \& Ryan, 2012).

Accordingly, with Deci and Ryan (2000), BPN are "innate psychological nutriments that are essential growth, integrity and well-being” (p. 229), for all individuals regardless of age, gender or culture (Ryan \& Deci, 2002). That is, these non-hierarchical needs are not "learnt", even considering that the means for meeting them may differ inside a specific culture or context (Chirkov, Ryan, Kim, \& Kaplan, 2003). Therefore, it is so important that questionnaires reflect the particular issues and cultural meanings which context language carries. In sum, the process of adapting instruments should consider the relevance of original instrument concepts and domains in the new context, considering the appropriateness of each item of the original instrument in terms of the ability to represent such concepts and domains in the new target population. Furthermore, the process should consider the semantic, linguistic, and contextual

\footnotetext{
Manuscript received at May 12 $2^{\text {th }} 2016$; Accepted at September $2^{\text {nd }} 2016$

${ }^{1}$ Escola Superior de Desporto de Rio Maior, ESDRM-IPSANTÁREM, Rio Maior, Portugal

${ }^{2}$ Universidade da Beira-Interior, UBI, Covilhã, Portugal

${ }^{3}$ Research Center in Sport, Health and Human Development, CIDESD, Portugal

* Corresponding author: Escola Superior de Desporto de Rio Maior, ESDRM-IPSANTÁREM), Av. Dr. Mário Soares, n. ${ }^{\circ} 110,2040-413$, Rio Maior E-mail: diogomonteiro@esdrm.ipsantarem.pt
} 
equivalence between the original and translated items and should include an analysis of the psychometric properties of the original instrument and its new version (Banville, Desrosiers, \& Genet-Volet, 2000; Vijver \& Hambleton, 1996).

Taking this in consideration, there has been some necessity for adapting and validate questionnaires to measure SDT constructs in the sports, including the evaluation of the BPN. For this reason, the researchers strategy to suppress the lack of specific instruments in the sports context involved using questionnaires from other domains, especially from the versions developed for work (Deci et al., 2001) and education contexts (Kowal \& Fortier, 2001). Besides this, other used strategies were the use of subscales and other questionnaires adapted to the different context within the studies of sports and physical activity (e.g., Bartholomew, Ntoumanis, Ryan \& Torgersen- Ntoumani, 2011; Gagné, Ryan \& Bargaman, 2003; Ntoumanis, 2001; Reinboth \& Duda, 2006; Sarrazin, Guillet, Vallerand, Pelletier \& Cury, 2002). However, under this issue, Gillet, Rosnet and Vallerand (2008) developed a scale in French (Échelle de Satisfaction des Besoins Fondamentaux en Context Sportif) with a sample of 236 athletes from several sports to assess the three basic psychological needs in the sport context. After analysing several works where the BPN had been studied in a sports context, the authors developed a scale that initially had 19 items.

Model fit was assessed using the chi-square value $\left(\chi^{2}\right)$, the Comparative Fit Index (CFI; Bentler, 1990), and the Root Mean Square Error of Approximation (RMSEA; Steiger \& Lind, 1980) accompanied by its $90 \%$ confidence interval $(90 \% \mathrm{CI})$

However, after an exploratory analysis, the scale was reduced to a final structure with three factors and 15 items, which demonstrated good adjustment to the data (i.e., Goodness Fit Index $=.92$; Non Normed Fit Index =.93; Comparative Fit Index $=.95$; Incremental Fit Index $=.95$; Standardized Root Mean Residual =.07; Root Mean Square Error of Approximation =.06). Yet, these adjustment levels were only achieved after the correlations of several errors of measure in the model (i.e., items $3-15,6-12$ and $6-15$ of the competence subscale; items 1-4, 7-10, 10-13 and $7-13$ of the autonomy subscale; and items 2-14 of the relatedness subscale), which is a practice that should be avoided (Marsh, Hau, \& Wen, 2004; Worthington \& Whittaker, 2006).

Later, Domínguez, Martín, Martín-Albo, Núnez, and Léon (2010) translated and validated the French version from Gillet et al. (2008) to Spanish on a sample of 282 federated athletes from several sports. However, the initial model did not adjust to the data in the first analysis (IFI=.87; CFI=.87; RMSEA=.09; RMSEA 90\% $\mathrm{CI}=.09-.10)$. Therefore, the authors followed the same strategy as Gillet et al. (2008), allowing the correlation of measure errors, namely from items 1 and 4 (from the autonomy subscale), after which the model adjusted itself to the data satisfactorily (IFI=.91; CFI=.91; $\mathrm{RMSEA}=.07$; RMSEA 90\% CI $=.06-.09$ ).

Furthermore, when analysing the standard parameters of the measured model (Domínguez et al., 2010, p. 1016), a low level of local adjustment was observed, namely on the factorial weight of items 1 and 4 (from the autonomy perception subscale) and item 6 (from the competence perception subscale), which showed values below the standard recommendations $(\lambda \geq .50)$. Consequently, these items did not explain at least $25 \%$ of the variance of the latent factor $\left(\lambda \mathrm{ij}^{2} \geq .25\right)$, as postulated by different authors (Byrne, 2010; Hair, Black, Babin, \& Anderson, 2014; Kline, 2011).

Based on the scientific evidence with poor quality of psychometric instruments that assess the BPN in the sport context, $\mathrm{Ng}$, Lonsdale and Hodge (2011) developed a scale to assess the underlying constructs of the SDT (i.e., Basic Needs Satisfaction in Sports Scale - BNSSS), which is comprised of five factors and 20 items. The scale was validated on a sample of 371 athletes from New Zealand, and demonstrated good adjustment to the data (i.e., NNFI=.96; $\mathrm{CFI}=.97 ; \quad \mathrm{SRMR}=.07 ; \quad \mathrm{RMSEA}=.06 ; \quad \mathrm{RMSEA}$ $90 \% \mathrm{CI}=05-.06)$. However, regarding the measure model underlying the BNSSS, it was advocated that the autonomy subscale as composed of three factors (i.e., autonomy for choice, internal perceived locus of causality and 
volition) and not only by one factor, as recommended by the SDT (Deci \& Ryan, 2008). This justified such decisions based on the opinion of a specialist group, which appreciated the content of the scale, arguing that there were certain aspects of the autonomy that were not reflected in the subscale (mentioned above) and recommended dividing the construct into three subscales (see study 2: Ng et al., 2011). However, in this same work, particularly study 1 (according to the theoretical model), the initial measure model, composed only of the three basic psychological needs (three factors, 15 items), presented a good adjustment to the data (i.e., $\mathrm{NNFI}=.98 ; \mathrm{CFI}=.98 ; \mathrm{SRMR}=.05$; $\mathrm{RMSEA}=.06$; RMSEA $90 \%=.04-.07)$ and good internal reliability for the autonomy subscale (.83), and did not present problems with the discriminant validity (Ng et al., 2011).

Briefly, it seemed that there was no general agreement in the literature on which scale was best suited to use for the assessment of the three BPN from SDT in the sport context. Due to the aforementioned shortages and necessities and based on the problems found in the French (Gillet et al., 2008) and Spanish scales (Domínguez et al., 2010), and the incompatibility between the measure model proposed by Ng et al. (2011) and the theoretical model underlying the SDT (Deci \& Ryan, 1985), a need for the existence and development of a sports context-specific questionnaire was felt.

The original version of the BPNES (Vlachopoulos \& Michailidou, 2006) was validated on a sample of Greek exercise participants in fitness centres, presented good adjustment values, and was translated and validated in other languages, namely English (Vlachopoulos, Ntoumanis, \& Smith, 2010), Portuguese (Moutão et al., 2012) and Spanish (Moreno-Murcia, Galindo, Pérez, Marcos, \& Borges, 2012), resulting in similar psychometric properties. This supports using the measurement model in different cultures, for which the scientific evidence was posteriorly confirmed in a transcultural study regarding the invariances of the measurement model of the BPNES between Greece, Turkey, Spain and Portugal (Vlachopoulos et al., 2013). Further, the
Portuguese version of the BPNESp was also adapted and validated preliminary for the physical education context (Pires, Cid, Borrego, Alves, \& Silva, 2010), whose model was confirmed in a transcultural study between Portugal and Brazil, where it supported the adequacy of the measurement model for these two countries, as well as their invariance (Cid et al., 2016). In addition to this evidence, some multi-sample studies over the past few years have shown that the basic psychological needs are equivalent across gender, i.e. they are experienced in the same way by men and women (Vlachopoulos, 2008), and also across community and private fitness centres exercise participants (Vlachopoulos, 2007).

For this reason, the aim of this study is to adapt and validate to sport context the Portuguese version of the Basic Psychological Needs Exercise Scale (BPNESp) (Moutão et al., 2012). In addition, considering that the generalizability of BPN theoretical tenets in the sports domain would require also expanding the evidence of item meaning equivalence across different sports, it is proposed to examine the extent of measurement invariance of the BPNES scores in the most representative collective and individual sports in Portugal, namely, football and swimming.

\section{Participants}

\section{METHODS}

The sample comprised 1382 federated athletes (1050 males, 323 females) from football (623) and swimming (759), with an average age of 18.28 ( $\mathrm{SD}=4.89$ years); all athletes practiced the sport at the national level. In the case of football, the national level is referred to the national leagues in a range of competition categories (i.e., initiates, juveniles, juniors and seniors) and in the case of the Swimming to the national championships of short and long courses. In this sample, the years of practice varied from 1 to 28 , (Mean=7.93; $\mathrm{SD}=2.80)$, the number of weekly trainings varied from 1 to 11 , and the duration of the training sessions from 60 to 180 minutes per day. The study was conducted on a sample of swimming and football athletes since these are the most representative collective and individual 
sports in Portugal (i.e. with more federate athletes), according to IPDJ (2016).

\section{Measures}

The Basic Psychological Needs in Exercise Scale (BPNESp: Moutão et al., 2012) was used. This questionnaire consisted of 12 items with a fivepoint Likert scale, which varied between 1 ("Strongly Disagree") and 5 ("Strongly Agree"). The items were grouped posteriorly into three factors (with four items each), which reflected the underlying basic psychological needs related to the SDT (Deci \& Ryan, 1985).

\section{Procedures}

\section{Data collection}

Every athlete and/or legal guardian was contacted individually by telephone in which, in addition to the explanation of the study's purposes, was requested an e-mail address to send the questionnaire. Each e-mail was sent, individually, with a different link for each subject, granting that they would receive the e-mail just once and a letter of intention, with the study purposes, rightly signed by all of its authors, in which the principle of confidentiality/anonymity was safeguarded. The questionnaires were filled through the survey monkey platform, with a mean filling time of 10 minutes.

\section{Adaption of the questionnaire to the sport domain}

The adaptation process of the questionnaire to the sport context was conducted by the researchers, who adjusted the exercise terms to the sport context without modifying their semantic content. For example, "I feel good with the people I exercise with" (exercise context) to "I feel good with my training friends" (sport context); or, "I feel that I successfully realize the activities from my exercise program" (exercise context) to, "I feel that I successfully realize the training activities" (sport context).

\section{Data Analysis}

Confirmatory Factor Analysis(CFA)

Regarding Confirmatory Factor Analysis (CFA), it was assumed a ratio of 15:1, in other words, fifteen subject's for each parameter to be estimated from the model, since the normalized coefficient of Mardia presented a non-normal multivariate distribution (47.1), as recommended by several authors (e.g., Byrne, 2010; Hair et al., 2014). Therefore, CFA was operationalized using maximum likelihood (ML), based on chi-square test $\left(\chi^{2}\right)$ and respective degrees of freedom (df), as well as the significance level (p).

In order to verify the quality assumptions of the measurement model adjustment, traditional absolute and incremental indexes were used: Standardized Root Mean Square Residual (SRMR), Root Mean Square Error of Approximation (RMSEA) and the respective confidence interval $(90 \% \mathrm{CI})$, Comparative Fit Index (CFI), Non-Normed Fit Index (NNFI), Root Mean, respectively. The most conservative cut-off values proposed by $\mathrm{Hu}$ and Bentler (1999) were adopted to these referred indexes: $\quad S R M R \leq .08, \quad C F I$ e $N N F I \geq .95$ e RMSEA $\leq .06$.

Additionally, the convergent validity was analysed (to check if the items were related to the respective factor) via the calculation of the average variance extracted (AVE), considering values of AVE $\geq .50$ (Fornell \& Larcker, 1981; Hair et al., 2014) and the composite reliability (CR) and Cronbach's Alpha was analysed to assess the internal consistency of the factors, adopting ( $\geq .70)$ as the cut-off values, as suggested by Hair et al. (2014).

\section{Multi-Group Analysis}

The multi-group analysis was conducted to assess whether the measurement model structure was equivalent (invariant) in different groups with different characteristics, in this case, between football and swimming sports. According to Byrne (2010) and Cheung and Rensvold (2002), in order to exist invariance it is necessary to verify two criteria's: a) the measurement model should be adjusted to each group; b) to perform a multigroup analysis, examining the following invariance types: configural invariance (i.e. unconstrained model); metric invariance (equal factor loadings/weak invariance); scalar invariance (i.e. strong invariance) and residual invariance (i.e. residual invariance of the items/strict invariance). According to Cheung and Rensvold (2002), the 
invariance assumptions are verified through the differences of $\chi^{2}$ test or CFI, and those should be $\triangle$ CFI $\leq .01$. The analysis was undertaken using AMOS 20.0.

\section{RESULTS}

As can be seen in Table 1, the individuals that used all answer levels (i.e., from 1 to 5) had higher means associated with items related to BPN. These answers also depicted a non-normal univariate distribution of the data, which presented a bias to the left, and could be explained by the tendency for the individuals to score in the highest levels of an answer (i.e., four and five) in this kind of Likert scale.

Regarding the model adjustment to the data, as it can be observed on table 2 , both model 1 (3factors/12 items) and model 2 ( 1 factor of $2^{\text {nd }}$ order; 3 factors of $1^{\text {st }}$ order and 12 items), adjusted in a sastisfatory way to the data in swimming and football, however, all the cut-off values adopted in the methodology ( $\mathrm{Hu} \&$ Bentler, 1999) were not reached. It is also possible to verify that the measurement model underlying to BPNESp (both exercise context and physical education adaptation) has presented evidences of its robustness.

Table 1

Descriptive analysis

\begin{tabular}{lcccccc}
\hline \multicolumn{1}{c}{ Item } & Min-Max & $\mathrm{M} \pm \mathrm{SD}$ & Skewness & $\mathrm{z}$ value & Kurtosis & $\mathrm{z}$ value \\
\hline Item 1 (Competence) & $1-5$ & $3.71 \pm 0.94$ & -0.67 & -10.27 & .339 & 2.56 \\
Item 2 (Relatedness) & $1-5$ & $3.96 \pm 1.00$ & -0.98 & -14.84 & .691 & 5.23 \\
Item 3 (Autonomy) & $1-5$ & $3.61 \pm 1.06$ & -0.66 & -.10 & -.033 & -2.50 \\
Item 4 (Competence) & $1-5$ & $3.87 \pm 0.90$ & -0.60 & -9.18 & .131 & .990 \\
Item 5 (Relatedness) & $1-5$ & $4.05 \pm 0.94$ & -1.00 & -15.25 & .925 & 7.01 \\
Item 6 (Autonomy) & $1-5$ & $3.11 \pm 1.10$ & -.10 & -1.61 & -.586 & -4.43 \\
Item 7 (Competence) & $1-5$ & $3.54 \pm 0.98$ & -.350 & -5.30 & -.305 & -2.31 \\
Item 8 (Relatedness) & $1-5$ & $3.96 \pm 1.05$ & -1.01 & -15.30 & .586 & 4.43 \\
Item 9 (Autonomy) & $1-5$ & $3.38 \pm 1.02$ & -.250 & -3.78 & -.357 & -2.70 \\
Item 10 (Competence) & $1-5$ & $4.03 \pm 0.82$ & -.690 & -10.45 & .502 & 3.80 \\
Item 11 (Relatedness) & $1-5$ & $4.05 \pm 0.95$ & -.996 & -15.09 & .861 & 6.52 \\
Item 12 (Autonomy) & $1-5$ & $3.26 \pm 1.06$ & -.262 & -3.96 & -.427 & -3.23 \\
\hline
\end{tabular}

Legend: M (Mean); SD (Standard Deviation)

Table 2

Goodness-of-fit indexes of the measurement model's (including existing versions)

\begin{tabular}{lcccccccc}
\multicolumn{1}{c}{ Models } & $\chi^{2}$ & $\mathrm{df}$ & $\mathrm{p}$ & SRMR & $\mathrm{NNFI}$ & $\mathrm{CFI}$ & $\mathrm{RMSEA}$ & $90 \%$ IC \\
\hline Greek Version $^{1}$ & 122.28 & 51 & .001 & .036 & .973 & .979 & .050 & $.041-.065$ \\
English Version $^{2}$ & $114.55^{*}$ & $41^{* *}$ & .001 & - & - & .948 & .073 & $.057-.089$ \\
Portuguese Version $^{3}$ & $144.14^{*}$ & 51 & .001 & - & .938 & .952 & .059 & $.048-.071$ \\
Spanish Version $^{4}$ & $161.67^{*}$ & 51 & .001 & .070 & .900 & .910 & .080 & - \\
Turkish Version $^{5}$ & $199.71^{*}$ & $42^{* *}$ & .001 & - & - & .912 & .074 & $.064-.084$ \\
Adaptation PE Portugal $^{6}$ & 203.8 & 51 & .001 & .062 & .926 & .943 & .070 & $.060-.080$ \\
Adaptation PE Brasil $^{6}$ & 173.7 & 51 & .001 & .052 & .940 & .954 & .073 & $.061-.085$ \\
Model 1 $_{\text {Model 2 }}^{435.20}$ & 51 & .001 & .049 & .932 & .945 & .074 & $.068-.080$ \\
Football Model $_{\text {Swimming Model }}$ & 435.20 & 51 & .001 & .049 & .936 & .949 & .074 & $.068-.080$ \\
\hline & 203.7 & 51 & .000 & .060 & .928 & .944 & .069 & $.060-.079$ \\
\hline
\end{tabular}

Legend: $\chi^{2}=$ chi-squared; $\mathrm{df}=$ degrees of freedom; SRMR = Standardized Root Mean Square Residual; NNFI = Non-Normed Fit Index; CFI = Comparative Fit Index; RMSEA = Root Mean Squared Error of Approximation; $90 \% \mathrm{CI}=$ confidence interval of RMSEA; ${ }^{1}$ Vlachopoulos et al. (2006); ${ }^{2}$ Vlachopoulos et al. (2010); ${ }^{3}$ Moutão et al. (2012); ${ }^{4}$ Moreno-Murcia et al. (2012); ${ }^{5}$ Vlachopoulos et al. (2013); ${ }^{6} \mathrm{Cid}$ et al. (2016); PE - Physical Education; Model 1 - Adaptation for the sport domain (three firstorder factors - autonomy, competence and relatedness, three factors/12 items); Model 2 - Adaptation for the sport domain (one second-order factor - global index of BPN, three first-order factors/12 items; * values reported by the authors concerning the Satorra-Bentler correction of $\chi^{2}\left(\mathrm{~S}-\mathrm{B}^{2}\right)$; ${ }^{* *}$ model with three factors and 11 items (in the case of the Turkish version with a correlation between the errors of measurement).

According to the results presented in Figure 1, a significant positive correlation existed between autonomy and competence $(\mathrm{r}=.59)$, competence and relatedness $(\mathrm{r}=.33)$ and autonomy to relatedness $(r=.29)$. Relative to the results of the adjustment of the model's individual parameters, factorial validity was present, i.e. all items had a factorial weight on the respective factor (all 
statistically significant; $\mathrm{p}<.05)$ varying from .62 to .73 for autonomy, .59 and .82 for competence and .73 and .91 for relatedness. Furthermore, all items explained more than $25 \%$ of the variance of the latent factor $\left(\lambda \mathrm{ij}{ }^{2} \geq .25\right)$, a value commonly accepted in the literature (Hair et al., 2014).

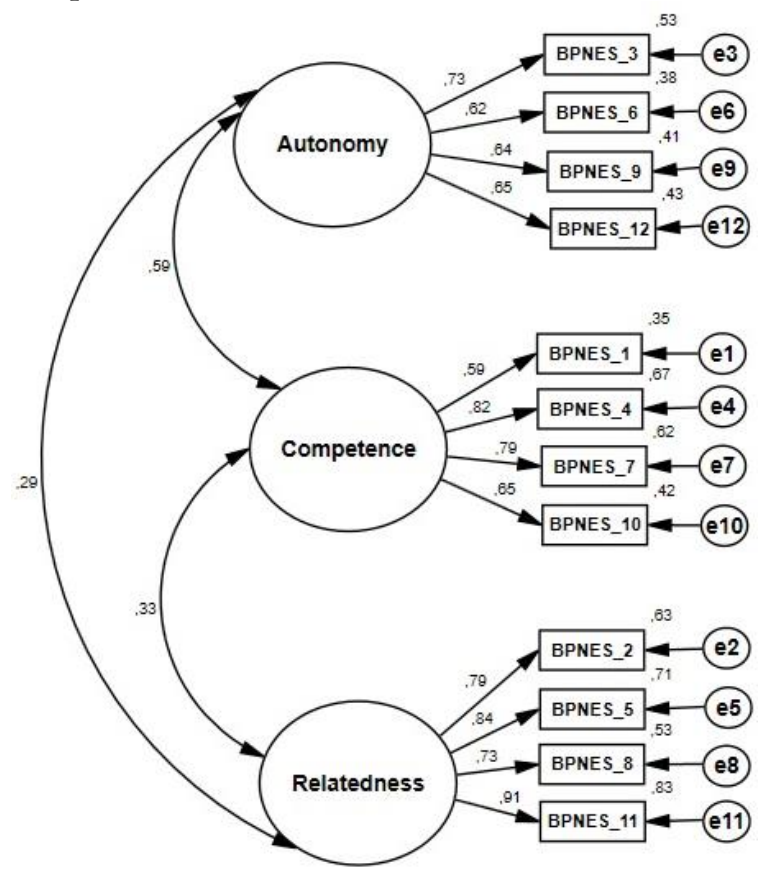

Figure 1: Standardized individual parameters for the adaptation to sport domain

Regarding the results presented in Figure 2, related to the values of adjustment of the secondorder model (i.e., one second-order factor; three first-order factors and 12 items), a significant positive effect existed $(\beta=.72 ; \beta=.82)$ between the second-order factor and autonomy and competence. In addition, a significant positive effect existed, which was moderated in total terms $(\beta=.40)$ between the second-order factor and relatedness.

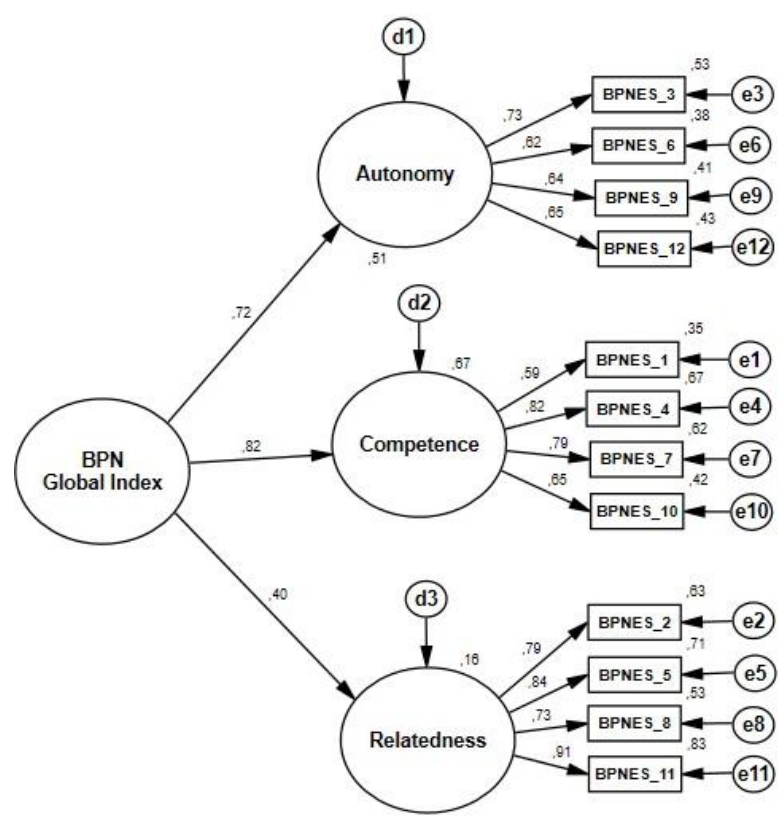

Figure 2: Standardized individual ( $2^{\text {nd }}$ order model) for the adaptation to sport domain

As seen in Table 3, the measurement model presented good values of internal consistency, both in terms of Cronbach's alpha reliability $(\alpha)$ and composite reliability (CR), as all values were $\geq .70$, as proposed by Hair et al. (2014). Regarding convergent validity, the autonomy factor presented, though with a minimum difference, an AVE value ( $\mathrm{AVE}_{\mathrm{Aut}}$ ) of .44, which was inferior to the recommended value (AVE $\geq .50$ ). The remaining factors presented adequate values of convergent validity ( $\mathrm{AVE}_{\mathrm{Comp}}=.51$; $\mathrm{AVE}_{\mathrm{Rel}}=.67$ ) (Hair et al., 2014). Still, none of the factors presented issues of discriminant validity, as the square of the factor's correlation was inferior to the AVE (Fornell \& Larcker, 1981; Hair et al., 2014).

Table 3

Internal reliability (with Cronbach's alpha in the diagonal), convergent and discriminant validity and average variance extracted

\begin{tabular}{cccccc}
\hline Factors & CR & AVE & Autonomy & Competence & Relatedness \\
\hline Autonomy & .75 & .44 & $\alpha=.76$ & & \\
Competence & .80 & .51 & $.34^{*}$ & $\alpha=.80$ & \\
Relatedness & .89 & .67 & $.08^{*}$ & $.10^{*}$ & $\alpha=.89$ \\
\hline Legend: Composite Reliability (CR); Average Variance Extracted (AVE); Cronbach's Alpha $(\alpha)$; $^{*}(\mathrm{r} 2)$ &
\end{tabular}

As it is shown on table 4 , the measurement model revealed to be invariant in function of the sports (football and swimming). Found values show the following aspects: configural invariance (i.e. unconstrained model), the same amount of manifest variables (i.e. items) is present in the amount of latent variables (i.e. factors); constrained model (i.e. metric invariance/weak invariance), factorial weights of respective factors (i.e. autonomy, competence and relatedness) from BPNESp adaptation related to sport context have the same meaning in both sports (football and swimming); scalar invariance (i.e. strong invariance), ensure that obtained results are 
totally related with individuals latent trait level, independently the group; residual invariance (i.e. residual invariance of the items), which confirms items residues are the same for the different groups. However, in this last case the assumption was not verified.

Table 4

Fit indexes for the invariance of the measurement model of the adaption of BPNES to a sport domain between football and swimming

\begin{tabular}{lccccccc}
\hline & $\chi^{2}$ & $\mathrm{df}$ & $\Delta \chi^{2}$ & $\Delta \mathrm{df}$ & $\mathrm{p}$ & $\mathrm{CFI}$ & $\Delta$ CFI \\
\hline Configural Invariance & 540.45 & 102 & - & - & - & .939 & - \\
Metric Invariance & 556.35 & 111 & 15.89 & 9 & .069 & .938 & .001 \\
Strong Invariance & 611.04 & 117 & 70.52 & 15 & .000 & .931 & .008 \\
Strict Invariance & 666.55 & 129 & 126.09 & 27 & .000 & .925 & .014
\end{tabular}

Legend: $\chi^{2}=$ chi-squared; $\mathrm{df}=$ degrees of freedom; $\Delta \chi^{2}=$ differences in the value of chi-squared; $\Delta d f=$ differences in the degrees of freedom; CFI = Comparative Fit Index; $\Delta \mathrm{CFI}=$ differences in the value of the Comparative Fit Index.

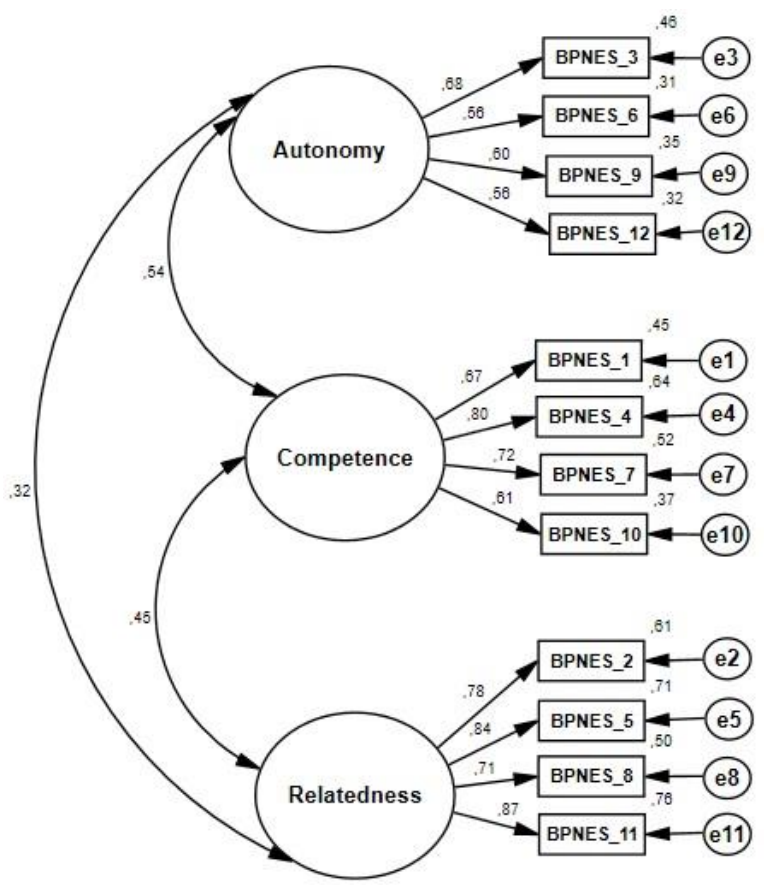

Figure 3: Standardized individual parameters (football sample)

As we may note from figures 3 and 4 , the standardized factorial weights of the items showed factorial validity, which means all items vary between .56 and .87 in the football sample, and between .58 and .93 in the swimming sample. Besides this, they all explain more than $25 \%$ of variability of the latent factor $\left(\lambda \mathrm{ij}{ }^{2} \geq .25\right)$ and were statistically significant $(\mathrm{p} \leq .05)$ in every factor. The two samples showed positive and significant correlations. The football sample had the following correlations: AutonomyCompetence $(\mathrm{r}=.54)$, Autonomy-Relatedness $(\mathrm{r}=.32)$ and Competence-Relatedness $(\mathrm{r}=.45)$. The swimming sample had the following correlations: Autonomy-Competence (.61) Autonomy-Relatedness (.29) and Competence-
Relatedness (.30). From the point of view of the internal consistency, both samples show a good composite reliability $(\mathrm{CR} \geq .70)$ in both factors. Football: $\quad($ CRaut $=.70 ; \quad$ CRCom $=.79$; CRRel $=89)$; $\quad$ Swimming: $\quad$ (CRaut $=.80$; CRCom=.81; CRRel $=89$ ).

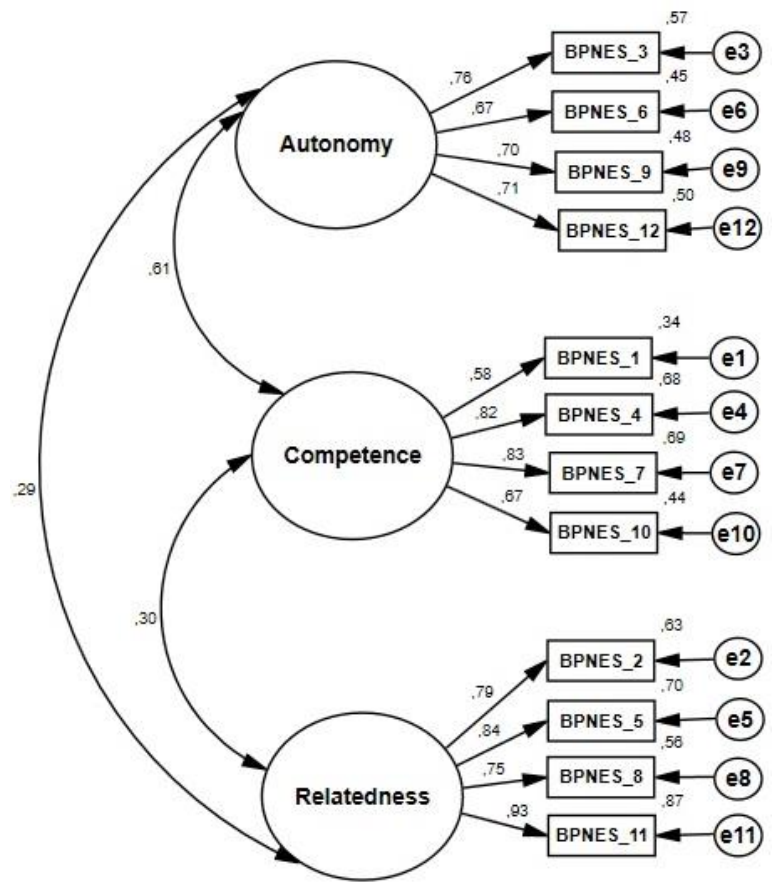

Figure 4: Standardized individual parameters (swimming sample)

\section{DISCUSSION}

The main purpose of this study was to analyse the psychometric qualities of the measurement model from the Portuguese version of the BPNESp (Moutão et al., 2012) adapted to the sport context. The process of adapting an existing instrument has considerable advantages, allowing the researcher to compare data from different samples and backgrounds with greater equality, since the same instrument assesses the 
construct based on the same theoretical and methodological perspectives. Therefore, an adapted instrument will have a greater ability to generalize the results and will also enable to investigate differences within an increasingly diverse population (Hambleton, 2005; Vlachopoulos et al., 2013), avoiding the chaotic proliferation of instruments that evaluate the same constructs. In addition, it is intended to analyse the model invariance between the football and swimming sports, enlarging the scientific evidence and contributing to what Deci and Ryan (2008) designated as knowledge development of the universality of the subjacent variables to the self-determination theory, which, in this concrete case, concerned the satisfaction of the BPN in the sport context.

In a descriptive way, the results revealed that the athletes tended to value the questionnaire items, which was demonstrated by the moderated and high means for all items, demonstrating the theoretical importance underlying to the three BPN and a tendency for the individuals to feel their BPN fulfilled in the sports context. These results are in line with the ones reported in different studies that used the BPNES, namely the original version (Vlachopoulos \& Michailidou, 2006), the Portuguese version (Moutão et al., 2012) and the Spanish version (Moreno-Murcia, Galindo, Pérez, Marcos, \& Borges, 2012). There were also positive correlation values and significant differences among the three factors (autonomy, competence and relatedness), which supports the evidence found in other studies (e.g., Moutão et al., 2012; Vlachopoulos, 2007; Vlachopoulos \& Michailidou, 2006).

Regarding the psychometric properties of the adaptation of the BPNESp to a sport context, the results, from both model 1 and model 2, demonstrated a reasonable adjustment to the data, showing its structure to be in line with the Portuguese version (Moutão et al., 2012) and the original version (Vlachopoulos \& Michilidou, 2006). Other studies used this scale with a Greek sample (Vlachopoulos, 2007, 2008; Vlachopoulos $\&$ Neikou, 2007), and the different versions of BPNES were used in the exercise context in England (Vlachopoulos et al., 2010), Spain
(Murcia et al., 2012) and Turkey (Vlachopoulos et al., 2013). Inclusively, the results from this study were similar to those of $\mathrm{Ng}$ et al. (2011), who used the scale specifically in the sport context with three factors and 15 items.

The results also showed that the measure model of the adapted version of the BPNES has an overall good quality of adjustment considering the model fit indexes values presented in table 2 (model 1, 2, football and swimming). These good psychometric properties support the validity of this questionnaire in the sport context, which, according to Hair et al. (2014), represent whether a group of items reflects the latent theoretical construct that it is supposed to measure. Although, it is important to state that these model fit indexes did not reach the more conservative cut-off values proposed by $\mathrm{Hu}$ and Bentler (1999), mentioned in the methodology section. Indeed, some authors (e.g., Byrne, 2010; Hair et al., 2014; Marsh et al., 2004), cautioned against dogmatic use of the cut-off values of $\mathrm{Hu}$ and Bentler (1999), since some of the characteristics of the data (e.g., sample size) and model complexity (e.g. number of variables) could account for some variance in fit indexes. Thus, in order not to reject good models, the mentioned authors recommend less conservative cut-off values, both in incremental indexes (i.e., CFI e TLI $\geq .90$ ) and in absolute indexes (SRMR e RMSEA 2.08) (Hair et al., 2014, Kline, 2011, Marsh et al., 2004). Regarding reliability, all factors revealed good internal consistency, with values of composite reliability and Cronbach alpha $\geq .70$ (Hair et al., 2014; Fornell \& Larcker, 1981).

The questionnaire did not show discriminant validity issues (see Table 3 ), as the results indicated that the defined factors for each group of items were distinct (Hair et al., 2014). Similar results were reported for the original (Vlachopoulos \& Michilidou, 2006), Spanish (Murcia et al., 2012) and Portuguese versions of the BPNES (Moutão et al., 2012). However, in the two latter cases, discriminant validity problems were verified between the constructs of autonomy and competence, which were justified by the high correlation between the two, as was observed in several studies (e.g., Vlachopoulos, 
2007, 2008; Vlachopoulos \& Michailidou, 2006). In addition, there was theoretical support for the greater autonomy perception of the individual, the greater the perception of competence, and vice-versa (Deci \& Ryan, 2000).

Regarding the convergent validity, competence and relatedness showed adequate values, namely (AVE $\geq .51$ and .67); however, the factor autonomy (AVE $\geq .50$ ), presented a minor issue, as it was close to the cut-off value (AVE=. 44), but all the factorial weights of this construct were equal to or greater than .62. Furthermore, Hair et al. (2014) suggested that if the factorial weights were all significant and greater than .50 , the factors would have good convergent validity, as occurred in the present study, and with the reported values of the Portuguese version of the BPNES (Moutão et al., 2012) from which this questionnaire was adapted.

Concerning the invariance of the measurement model between football and swimming, the results found allow us to claim that the measurement model underlying to the adaptation of BPNESp to the sport context is equivalent both in swimming and football, which means the basic psychological needs are perceived in the same way by the athletes of both sports.

This way, based on the assumptions of the model's invariances analysis, operationalized in methodology, we can claim that both in Swimming and in Football the following: that the same set of items that explain the same set of factors is kept, independently of the practiced modality, which confirms the configurational invariance; all factorial weights are invariant in both sports. This shows that items reveal the same importance for the factors, no matter what the modality is, thus providing metric invariance; the intercepts of the items are equivalent in both sports. According to Chen (2008), when this assumption is verified (i.e. strong invariance), it means it is legitimate to make result comparisons in different groups, in this case between football players and swimmers, is based on the theoretical assumptions of SDT.

According to Chen (2008), when this assumption is not verified the differences found among groups may be related not with the real differences at the level of latent variables, but with the non-equivalence of the instrument patterns. About the residual invariance, this assumption is not verified. However, according with several authors (e.g., Byrne, 2010; Cheung and Rensvold, 2002; $\mathrm{Wu}, \mathrm{Li}$, and Zumbo, 2007), there seems to be no consensus in literature about the need to evaluate residual invariance, for the reason that the evaluation of this assumption is considered optional by the investigator, due to the fact that it is too restrictive and difficult to achieve in investigations in the area of social sciences, which does not mean by that the lack of invariance of the measurement model (Byrne, 2010).

Similar results were found in the exercise context, (Vlachopulos et al., 2013), in physical education (Cid et al., 2016) and among genders in several studies (e.g., Vlachopoulos, 2008). These results reveal not only the quality of this measurement model in the evaluation of the three basic psychological needs, but also support what Deci and Ryan (2000) have said, which is that the basic psychological needs are innate and universal to human beings, whatever gender, ethnicity or cultural repertoire.

\section{CONCLUSIONS}

According to the results, the measurement model's three factors and 12 items, as well as the second-order model one factor (three first-order factors and 12 items of the BPNESp adaptation for the sport context) had acceptable psychometric properties. Regarding the second order tested model (one second-order factor; three first-order factors and 12 items), the evidence supported empirically its sustention (Moutão et al., 2012; Vlachopoulos, 2007). The analysis of the invariance of the measurement model revealed that it can be used with high validity and reliability in Swimming and Football. Lastly, and sharing Barret's (2007) opinion who stated that the evaluation of a model was always a process with countless obstacles, which required a great amount of time. Thus, we advise that future studies use this scale in other sports and that invariance analysis is used with this model of measurement on other variables or cultures, thus creating an even stronger model 
from a psychometric point of view. Finally, it is also important to highlight that now the scale is available as a Portuguese measurement instrument that assesses BPN underlying to the SDT (Deci \& Ryan, 2008) in a sport context, thus filling an existing lack.

\section{Acknowledgments:}

Nothing to declare

\section{Conflict of Interests:}

Nothing to declare

\section{Funding:}

Nothing to declare

\section{REFERÊNCIAS}

Banville, D., Desrosiers, P., \& Genet-Volet, Y. (2000). Translating Questionnaires and Inventories Using a Cross-Cultural Translation Technique. Journal of Teaching in Physical Education, 19, 374387.

Barret, P. (2007). Structural equation modelling: Adjudging model fit. Personality and Individual Differences, $\quad 42, \quad 815-824$. doi:10.1016/j.paid.2006.09.018

Bartholomew, K., Ntoumanis, N., Ryan, R., \& Thorgersen-Ntoumani, C. (2011). Psychological needs thwarting in sport context: Assessing the darker side of athletic experience. Journal of Sport \& Exercise Psychology, 33, 75-102. doi:10.1016/j.psychsport.2013.03.007

Byrne, B. (2010). Structural Equation Modeling with EQS. Basic Concepts, Applications, and Programming $\left(2^{\text {nd }}\right.$ ed.). Mahwah, NJ: Lawrence Erlbaum Associates, Publishers.

Chen, F. (2008). What happens if we compare chopsticks with forks? The impact of making inappropriate comparisons in cross-cultural research. Journal of Personality and Social Psychology, 95(5), 1005-1018. doi: 10.1037/a0013193

Cheung, G., \& Rensvold, R. (2002). Evaluating goodness-of-fit indexes for testing measurement invariance. Structural Equation Modelling: A Multidisciplinary Journal, 9(2), 233-255. doi:10.1207/S15328007SEM0902 5

Chirkov, V., Ryan, R., Kim, Y., \& Kaplan, U. (2003). Differentiating autonomy from individualism and independence: A self-determination perspective on internalization of cultural orientations, gender and well-being. Journal of Personality and Social Psychology, 84, 97-110. doi:10.1037//00223514.84.1.97

Cid, L., Stobaus, C., Monteiro, D., Moutão, J., Davoglio, T., \& Lettnin, C. (2016). Cross-cultural validation of the basic psychological needs in physical education between Portugal and Brazil
Samples. Spanish Journal of Psychology, 19(5). doi: 10.1017/sjp.2016.6

Deci, E., \& Ryan, R. (1985). Intrinsic Motivation and SelfDetermination in Human Behavior. New York, NY: Plenum Press. doi:10.1007/978-1-4899-2271-7

Deci, E., \& Ryan, R. (2008). Facilitating optimal motivation and psychological well-being across life's domains. Canadian Psychology, 49, 14-23. doi:10.1037/0708-5591.49.1.14

Deci, E. \& Ryan, R. (2000). The "what" and "why" of goal pursuits: Human needs and the selfdetermination of behavior. Psychological Inquiry, $11(4)$, 227-268. doi:10.1207/S15327965PLI1104_01

Deci, E., Ryan, R., Gagné, M., Leone, D., Usunov, J., \& Kornazheva, B. (2001). Need satisfaction, motivation, and well-being in the work organizations of a former eastern bloc country: A cross-cultural study of self-determination. Personality and Social Psychology Bulletin, 27(8), 930-942. doi:10.1177/0146167201278002

Domínguez, E., Martín, P., Martín-Albo, J., Núñez, J., \& Léon, J. (2010). Translation and validation of the Spanish version of the "Échelle de Satisfaction des Besoins Psychologiques" in the sports context. Spanish Journal of Psychology, 13 (2), 10101020. doi:10.1017/S1138741600002651

Fornell, C. ,\& Larcker, D. F. (1981). Evaluating structural equation models with unobservable variables and measurement error. Journal of Marketing Research, 18(1), 39-50. doi: $10.2307 / 3151312$

Gagné, M., Ryan, R., \& Bargmann, K. (2003). Autonomy support and need satisfaction in the motivation and well-being of gymnasts. Journal of Applied Sport Psychology, 15, 372-390. doi: $10.1080 / 10413200390238031$

Gillet, N., Rosnet, E., \& Vallerand, R. (2008). Développment d'une échelle de satisfaction des besoins fondamentaux en context sportif. Canadian Journal of Behavioural Science, 40(4), 230237. doi:10.1037/a0013201

Hair, J., Black, W., Babin, B., \& Anderson, R. (2014). Multivariate Data Analysis ( $7^{\text {th }}$ ed.). New Jersey: Pearson Educational, Inc.

Hambleton, R. K. (2005). Issues, designs, and technical guidelines for adapting tests into multiple languages and cultures. In R. K. Hambleton, P. F. Merenda, \& C. D. Spielberger (Eds.), Adapting educational and psychological tests for cross-cultural assessment (pp. 3-38). Mahwah, NJ: Lawrence Erlbaum.

Hu, L., \& Bentler, P. (1999). Cut off criteria for fit indexes in covariance structure analysis: Conventional criteria versus new alternatives. Structural Equation Modeling, 6(1), 1-55. doi:10.1080/10705519909540118

IPDJ (2016). Praticantes desportivos federados: total e por algumas federações desportivas. Available in: http://www.pordata.pt/Portugal/Praticantes + de sportivos + federados + total $+\mathrm{e}+$ por + algumas $+\mathrm{f}$ edera\%C3\%A7\%C3\%B5es+desportivas-2226. 
Kline, R. (2011). Principles and Practice of Structural Equation Modeling ( $3^{\text {rd }}$ ed.). New York, NY: The Guilford Press.

Kowal, J., \& Fortier, M. (2000). Testing relationship form hierarchical model of intrinsic and extrinsic motivation using flow as a motivational consequence. Research Quarterly for Exercise and Sport, 71, 171-181. doi:10.1080/02701367.2000.10608895

Mardia, K. (1970). Measures of multivariate skewness and kurtosis. Biometrika, 57(3), 519-530. http://dx.doi.org/ doi: 10.1093/biomet/57.3.519

Marsh, H. (1993). The multidimensional structure of physical fitness: Invariance over gender and age. Research Quarterly for Exercise and Sport, 64, 256273. doi:10.1080/02701367.1993.10608810

Marsh, H., Hau, K., \& Wen, Z. (2004). In search of golden rules: Comment on hypothesis testing approaches to setting cutoff values for fit indexes and dangers in overgeneralizing $\mathrm{Hu}$ and Bentler's (1999) findings. Structural Equation Modeling, $11(3)$, 320-341. doi:10.1207/s15328007sem1103 2

Moreno-Murcia, J., Galindo, C., Pérez, V., Marcos, P., \& Borges, F. (2012). Confirmation of the Basic Psychological Needs in Exercise Scale (BPNES) with a sample who do healthy exercise. Journal of Sports Science and Medicine, 11(11), 141-146.

Moutão, J., Cid, L., Leitão, J., Alves, J., \& Vlachopoulos, S. (2012). Validation of the Basic Psychological Needs in Exercise Scale in a Portuguese Sample. Spanish Journal of Psychology 15(1), 399-409. doi:10.5209/rev SJOP.2012.v15.n1.37346

Ng, J., Londsdale, C., \& Hodge, K. (2011). The Basic Needs Satisfaction in Sport Scale (BNSSS): Instrument development and initial validity evidence. Psychology of Sport and Exercise, 12, 257264. doi:10.1016/j.psychsport.2010.10.006

Ntoumanis, N. (2001). A self-determination approach to the understanding of motivation in physical education. British Journal of Educational Psychology, 71, 225-242. doi:10.1348/000709901158497

Pires, A., Cid, L., Borrego, C., Alves, J., \& Silva, C. (2010). Preliminary validation of a questionnaire to measure basic psychological needs in physical education. Motricidade, 6(1), 33-51. doi:10.6063/motricidade.6(1).157

Reinboth, M., \& Duda, J. (2006). Perceived motivational climate, need satisfaction and indices of well-being in team sports: A longitudinal perspective. Psychology of Sport and Exercise, 7(3), 269-288. doi:10.1016/j.psychsport.2005.06.002

Sarrazin, P., Vallerand, R., Guillet, E., Pelletier, L., \& Cury, F. (2002). Motivation and dropout in female handballers: A 21-month prospective study. European Journal of Social Psychology, 32, 395-418. doi:10.1002/ejsp.9

Teixeira, P., Carraça, E., Markland, D., Silva, M., \& Ryan, R. (2012). Exercise, physical activity, and self-determination theory: A systematic review. International Journal of Behavioral Nutrition and Physical Activity, 9, 78. doi:10.1186/1479-5868-978

Vijver, F., \& Hambleton, R. (1996). Translating Tests. Some Practical Guidelines. European Psychologist, 1(2), 89-99.

Vlachopoulos, S. (2008). The Basic Psychological Needs in Exercise Scale: Measurement invariance over gender. Structural Equation Modeling: A Multidisciplinary Journal, 15, 114-135. doi:10.1080/10705510701758398

Vlachopoulos, S. (2007). Psychometric evaluation of the Basic Psychological Needs in Exercise Scale in community exercise programs: A cross-validation approach. Hellenic Journal of Psychology, 4, 52-74.

Vlachopoulos, S., Asci, H., Cid, L., Ersoz, G., GonzálezCutre, D., Moreno-Murcia, J., \& Moutão, J. (2013). Cross-cultural invariance of the Basic Psychological Needs in Exercise Scale and latent mean differences among Greek, Spanish, Portuguese, and Turkish samples. Psychology of Exercise and Sport, 14(5), 622-631. doi:10.1016/j.psychsport.2013.03.002

Vlachopoulos, S., \& Michailidou, S. (2006). Development and initial validation of a measure of autonomy, competence and relatedness in exercise: The Basic Psychological Needs in Exercise Scale. Measurement in Physical Education and Exercise Science, 10, 179-201. doi:10.1207/ s15327841mpee1003_4

Vlachopoulos, S., \& Neikou, E. (2007). A prospective study of the relationships of autonomy, competence, and relatedness with exercise attendance, adherence, and dropout. Journal of Sports Medicine and Physical Fitness, 47, 475-482.

Vlachopoulos, S., Ntoumanis, N., \& Smith, L. (2010). The Basic Psychological Needs in Exercise Scale: Translation and evidence for cross-cultural validity. International Journal of Sport \& Exercise Psychology, 8, 394-412. doi: 10.1080/1612197X.2010.9671960

Worthington, R., \& Whittaker, T. (2006). Scale development research. A content analysis and recommendations for best practices. The Counselling Psychologist, 34(6), 806-838. doi:10.1177/00110000006288127

Wu, A., Li, Z., \& Zumbo, D. (2007). Decoding the meaning of factorial invariance and updating the practice of multi-group confirmatory factor analysis: a demonstration with TIMSS data. Practical Assessment, Research \& Evaluation, 12(3), $1-26$. 